\title{
Isomeric Xylene molecules in the terahertz-far infrared regime: computational chemistry and spectral modeling view
}

\author{
Annika Bande ${ }^{\mathrm{a}, 1}$, Ivona Bravića ${ }^{\text {, Clemens B. Minnich }}{ }^{\mathrm{b}}$, Ljiljana Puskar ${ }^{\mathrm{a}}$, Eglof Ritter ${ }^{\mathrm{c}}$, Ulrich \\ Schade ${ }^{\text {a }}$ Emad F. Aziz ${ }^{\text {a,d,e }}$ \\ ${ }^{a}$ Institute of Methods for Material Development, Helmholtz-Zentrum Berlin für Materialien und Energie GmbH, \\ Albert-Einstein-Straße 15, 12489 Berlin, Germany \\ ${ }^{b}$ S-PACT GmbH, Burtscheider Str. 1, 52064 Aachen, Germany \\ 'Institute of Biology, Humboldt-University, Invalidenstr. 42, 10115 Berlin, Germany \\ ${ }^{d}$ Department of Physics, Freie Universität Berlin, Arnimallee 14, 14195 Berlin, Germany \\ ${ }^{e}$ School of Chemistry, Monash University, Clayton 3800, VIC, Australia
}

Date: $2017-06-20$

\begin{abstract}
The theoretical assignments of spectral peaks of liquid phase ortho-, meta-, and para-xylene recorded with far-infrared (FIR) and THz spectroscopy in the spectral range between $50 \mathrm{~cm}^{-1}$ and $550 \mathrm{~cm}^{-1}$ is done with density functional theory (DFT) calculations. As THz spectroscopic techniques drastically evolved in recent years, the critical focus of this paper lies on the applicability of theoretical concepts, used as computational standard in near- and mid-IR spectra, to the FIR-THz region. An evaluation of the choice of functionals, basis sets, and appropriate scaling factors as well as the tractability of the liquid phase in a polarizable continuum model is performed. Alongside a new analysis procedure based on spectral Hard Modeling has been developed. DFT line spectra are fitted to experimental FIR spectra where a quantitative track record allows for meaningful comparisons. With all these tools we are able to reproduce experimental spectra in an optically appealing way and we can explain trends for each spectrum as well as across the row of the isomers.
\end{abstract}

Keywords:

density-functional theory; far-infrared; spectral Hard Model; xylene

${ }^{1}$ Corresponding author: annika.bande@helmholtz-berlin.de 


\section{Introduction}

Spectroscopic investigations, ideally supported by theoretical calculations, reveal various properties of the compound under study. The assignment of diverse functional groups to a molecule through detection of their vibrations is done with infrared spectroscopy, typically at wavenumbers 400 $4000 \mathrm{~cm}^{-1}$, i.e. in the mid-infrared (MIR). Nowadays a catalog of characteristic bands exists in that energy range for various types of vibrations of diverse functional groups that can be used to assign IR spectra [1].

The far-infrared (FIR) region of the electromagnetic spectrum is also relevant for molecular spectroscopy. A few FIR absorption spectra of common chemicals together with the assignment of their vibrational features are dating back to the 60 s and 70 s of the last century as, for example, in the case for the xylene isomers [2]. Over the last two decades FIR spectroscopy experienced a renaissance by the tremendous development of $\mathrm{THz}$ technology [3] leading to applications as manifold as for the MIR region. The most targeted molecules by modern spectroscopic and computer techniques turned out to be small biomolecules and peptides $[4,5,6,7,8,9,10,11]$.

Observables in spectroscopy, i.e. the interaction of molecules with light, more than in any other discipline in chemistry are connected to the quantum structure of a molecule. Hence, theoretical calculation make a very important contribution to the interpretation of spectra. Theoretical IR spectroscopy dates back to the discovery of the Schrödinger equation, when the quantum motion of a Hook's law spring reflecting a vibrating diatomic molecule was solved analytically. With the extension to many-atom molecules and methods to approximately solve quantum mechanical equations a hand-in-hand development of IR theory and experiment was possible.

Two major theoretical routes are available to predict vibrational spectra. In real-time nucleardynamics approaches as e.g. ab initio molecular dynamics [12], multidimensional quantum dynamics [13], or vibrational quantum Monte Carlo methods [14], the vibrations of a molecule are calculated in the time-domain and spectra are then deduced from the Fourier transformation of the changes of the time-dependent wave function. Using these approaches requires typically extensive theoretical derivation prior to computationally demanding calculations so that the established theoretical standard is ab initio electronic structure theory available at lower computational cost, which is straightforwardly usable from a number of widespread program packages. Here, highly correlated wave function based methods such as configuration interaction or coupled cluster that give highly accurate results may be used for small molecules. The most widespread intermediately accurate 
alternative which is technologically applicable to even relatively large compounds in various phases is density functional theory (DFT), which we use in this work.

DFT and other $a b$ initio calculations yield all possible fundamental vibrations in the mid- and in the far-infrared regime. Due to the much more widespread use of MIR in analytics, the quality of calculations has been proven in numerous examples for that energy range. There is also a number of recent studies in the FIR including theoretical investigations which report on the generally good quality of DFT calculations in that energy range $[4,6,7,8,10,11]$. However, only few of them even mention the necessity for a treatment of solvent effects and scaling of frequencies in liquid [4] or crystalline samples $[5,8]$ or comment on the reliability of the calculation of intensities compared to frequencies [5].

This paper deals with the assignment of the far-infrared absorption bands of the recently measured neat liquids ortho-, meta-, and para-xylene [15] for which we validate in detail the use of widespread DFT in FIR. We discuss explicitly the treatment of the liquid phase in a theoretical solvation model and the scaling of vibrational frequencies. We, moreover, develop a means for a quantitative comparison among theoretical and experimental spectra by fitting theoretical spectral lines with the spectral Hard Modeling approach known for the interpretation of experimental spectra of mixtures to the experimental spectrum [16].

The experimental xylene spectra have been taken from an FIR study on hazardous liquids $[15,17,18]$. The liquids have been investigated by spectroscopic ellipsometry in attenuated total reflection (ATR) geometry, a technique not available for gas phase measurements. In contrast to conventional transmission measurements using IR transparent cuvettes this experimental approach provided us with a Kramers-Kronig consistent pair of material parameters from which we directly obtained the spectral extinction coefficient, k, for our study. Among the characterized liquids were the benzene derivatives ortho-, meta-, and para-xylene whose spectra have been compared with less accurate and less sensitive measurements from 1962 [2].

This paper is organized as follows: Sec. 2 gives the introduction of the theoretical background on quantum mechanics (2.1) and the spectral Hard Model (2.2), whereas sections 3.1 and 3.2 comprise the respective computational details. The results and discussion section contains aspects on the choice of density functionals (4.1), the treatment of the liquid phase (4.2), and the use of scaling factors for wavenumbers (4.3). After this we switch to the findings on the xylenes themselves regarding their geometries (4.4), spectra (4.5), and trends of vibrational properties among the group of molecules (4.6). All of this is summarized in the conclusions (Sec. 5). 


\section{Theory}

\subsection{Computational Chemistry}

Quantum-mechanical calculations of vibrational spectra are performed on the equilibrium geometry $\vec{R}=\vec{R}_{e q}$ of a molecule in its electronic ground state i.e. in the minimum $V_{0}$ of the potential $V(\vec{R})$. Any displacement of atoms along the normal modes increases $V(\vec{R})$ which is approximately given through an $n$-dimensional harmonic oscillator equation

$$
V(\vec{R})=V_{0}+\left.0.5 \sum_{i=1}^{3 \mathrm{~N}-6} \sum_{j=1}^{3 \mathrm{~N}-6} \frac{\partial^{2} V}{\partial \vec{R}_{i} \partial \vec{R}_{j}}\right|_{\vec{R}_{e q}}\left(\vec{R}_{i}-\vec{R}_{e q}\right)\left(\vec{R}_{j}-\vec{R}_{e q}\right)
$$

The square matrix of the second derivatives of the potential which has the dimensionality of all coordinate displacements $\vec{R}_{i}$ is termed the Hessian matrix or, if mass weighted, the force constant matrix

$$
F_{i j}=\left.\left(m_{i} m_{j}\right)^{-1 / 2} \frac{\partial^{2} V}{\partial \vec{R}_{i} \partial \vec{R}_{j}}\right|_{\vec{R}_{e q}}
$$

Its eigenvalues $\lambda_{n}$ for any normal vibrational mode convert straightforwardly to the normal mode frequencies

$$
v_{n}=\sqrt{\lambda_{n}} / 2 \pi
$$

and hence to the wavenumbers $\tilde{v}_{n}$ of discrete vibrational states in the calculated line spectrum.

Corresponding line intensities $T_{n}{ }^{2}$, which are proportional to the oscillator strength, are obtained when the first and second vibrational wave functions $\psi_{0}^{\text {vib }}$ and $\psi_{1}^{\text {vib }}$ are explicitly known, hence one needs to fully solve the nuclear Schrödinger equation for a vibration using the potential of Eq. (1). Then $T_{n}^{2}$ is calculated as the squared expectation value of the dipole moment changing with all atomic displacements $\vec{R}_{i}$ unified in the normal coordinate $\vec{Q}_{n}$ for the fundamental $0 \rightarrow 1$ transition [19,20]:

$$
T_{n}^{2}=\left|\int \psi_{0}^{v i b}\left(\frac{\partial \hat{\mu}_{N}}{\partial \vec{Q}_{n}}\right) \vec{Q}_{n} \psi_{1}^{v i b} d \vec{Q}_{n}^{2}\right|^{2}
$$

Contrary to a theoretical spectrum, the measured spectral extinction coefficient displays a convoluted band spectrum due to Doppler broadening, radiation damping, and collision broadening. For a band 
of the mode $n$, which is assumed to be clearly separated energetically from other bands, the band area $A_{n}$ is obtained from the integration over the extinction coefficient $k(\tilde{v})$, i.e.

$$
A_{n}=\int_{\text {Band }} k(\tilde{\mathrm{v}}) d \tilde{\mathrm{v}}
$$

$A_{n}$ is a measure of the intensity of a vibrational transition. In the case of several overlying bands those are deconvoluted in a peak-picking approach by fitting several Gaussian, Voigt, or Lorentzian functions to the respective peak. Integrating individually over the fit functions the $A_{n}$ are determined and the wavenumbers $\tilde{v}_{n}$ are read from the positions of the maxima.

The quality of peak positions $\tilde{v}_{n}$ depends on the theoretical method chosen. The well-studied deficiencies e.g. of DFT methods in combination with a specific basis set requires the application of an empirically based scaling routine to shift calculated peaks towards corrected positions prior to comparison with experiment [21].

For the numerical comparison of experimental and theoretical spectra, $A_{n}$ and $\mathrm{T}_{n}{ }^{2}$ can actually both be linearly converted into the same oscillator strength, or relative intensities $I_{\text {rel }}=T^{2} / T^{2}{ }_{\max }=A / A_{\max }$ are compared. For a more common visual comparison theoretical peaks are typically simply overlaid with the best fitting Gaussian or Lorentzian curves of constant widths in the order of $5 \mathrm{~cm}^{-1}$ that accounts for peak broadening in the experiment.

Once the peak assignment has been finished the understanding and interpretation of spectra are facilitated as the calculations provide a visualization of all vibrational motions.

\subsection{Spectral Hard Modeling}

For the validation of quantum-mechanically calculated infrared data with the experimentally recorded spectra, an approach of spectral Hard Modeling was applied. Amongst the related methods it has found the widest applicability for predicting concentrations from spectra of mixtures [16]. Nevertheless, the structure of this Hard Model is expected to be equally valuable for the reported work to compare measured pure component spectra and their calculated counterparts.

The spectral Hard Model is a mathematical representation of a spectrum derived from fundamental physics: the model $S$ of a mixture spectrum is composed of $k$ peaks originating from the $i$ different components in the mixture. This structural information is maintained in the Hard Model

$$
S=\sum_{i} w_{i} \sum_{k} V_{i, k}(\tilde{\mathbf{v}})
$$


a sum of peak-shaped curves

$$
V(\tilde{v})=\beta \alpha \exp \left[\frac{-4 \ln 2(\tilde{v}-\omega)^{2}}{\gamma^{2}}\right]+(1-\beta) \alpha \frac{\gamma^{2}}{(\tilde{v}-\omega)^{2}+\gamma^{2}}
$$

weighted by the component weight $w_{i}$. For readability we neglected in Eq. (7) the indices $k$ on all peak parameters $\theta=\{\alpha, \beta, \gamma\}$ and the location of the peak maximum (peak position) $\omega$. $V$ is typically of (pseudo-)Voigt type, which means a sum of a Gaussian Part (left term) contributing with $0 \leq \beta \leq 1$ and a Lorentzian part (right term). Moreover, $\alpha$ is the peak amplitude and $\gamma$ the half width at half maximum (HWHM).

The $i$ subsets of $k$ peak-curves representing the pure component spectra are referred to as Component Models. Since all model parameters are physical quantities, the parametrization of the model can account for discrepancies between calculations (Secs. 2.1. and 3.1.) and measurements [15], i.e. scaling, peak shifts or peak shape changes. Typically, the spectral Hard Model for a mixture is obtained by merging component models that were derived from the respective pure component spectra by means of auto-fitting the constituent peaks [22].

When fitting a spectral Hard Model to an experimental spectrum, the spectral residuum over the entire spectral range is minimized by a least-squares approach. Unlike Classical Least-Squares approaches for spectral analysis, spectral Hard Models bear the flexibility to account for nonidealities like peak shifts or shape changes by automated manipulation of the peak-position and peak-shape parameters $\theta$. Relative peak intensities within a component model can be forced to be constant by a constant-peak-area boundary condition. Consequently, the overall appearance of a component spectrum will then be maintained, and only the scaling factor of the entire component model (- the scalar "component weight" $\left.w_{i}\right)$ - carries the quantitative information useful for the prediction of concentrations [23].

The novelty of the presented work lies in using the Hard Modeling approach for fitting calculated spectra to experimentally obtained ones. Therefore, the calculated line spectra are converted into a Hard Model S (Eq. (6)) of only a single component $i$ and fitted as such. As the calculations are based merely on ideality assumptions, the result of the Hard Model fit is expected to represent nonidealities in the experimental spectrum, including peak shifts (position changes) or the degree of peak deformation (shape changes) e.g. through molecular interactions. The application of a Hard Model fit to deconvolve the measured spectrum is beneficial over a simple peak picking approach 
which locally identifies peak-shaped maxima in the spectrum. Only in the Hard Model approach the overall peak pattern can be maintained, especially the relative intensities.

\section{Computations}

\subsection{Computational Chemistry}

The optimized gas and liquid phase geometries of ortho-, meta-, and para-xylene as well as their theoretical vibrational spectra have been obtained from density functional theory calculations as implemented in the quantum chemistry software package ORCA [24]. A few functionals of different levels of integrity, namely the GGA functional BP86 [25,26,27], the hybrid functionals PW6B95 [28] and TPSSO [29], and the double-hybrid functional B2PLYP [30] that had been reported to be well suited for the determination of properties [31] and vibrations [32] of organic molecules, have been tested for the three xylenes. The basis sets used were TZVP [33] and def2-TZVP $[33,34]$ as a quality below triple-zeta is considered to be insufficient and calculations with that basis set size are affordable for the small-sized target molecules. We made use of the RI approximation $[35,36,37,38]$ together with consistent auxiliary basis sets [39] which is for all hybrid functionals realized through the RIJCOSX formalism [40,41,42]. Furthermore, we used the D3 dispersion correction $[43,44]$. Besides functionals and basis sets we finally compared the performance of tight convergence criteria for the self-consistent field and the geometry together with a denser integration grid against a less dense grid in combination with the looser default convergence criteria. As measurements have been done in the liquid phase of the respective pure xylene we finally benchmark the use of the continuum solvation model (COSMO) implemented in ORCA [45]. For $o^{-}, m-$, or $p$-xylene the dielectric constants $2.57,2.374$, and 2.2 as well as the refractive indices $1.5054,1.49722$, and 1.4958 were applied.

The wavenumbers $\tilde{v}_{n}$ of the harmonic normal modes in $\mathrm{cm}^{-1}$ obtained from the frequency calculations were scaled with empirical factors for the respective method and basis set to account for the anharmonicity and ensemble effects so that they best reproduce the experimentally obtained fundamental frequencies. Scaling factors used here in combination with the def2-TZVP basis set were 0.9953 for BP86, 0.9505 for TPSSO, and 0.9623 for B2PLYP [32]. No scaling factor is published for PW6B95 with the def2-TZVP basis set, therefore we used 0.945 for the $6-31+G(d, p)$ basis instead [46]. 


\subsection{Spectral Hard Modeling}

For spectral Hard Modeling we made use of the IHM implementation in the analysis software PEAXACT (version 4.0, S-PACT GmbH). All experimental spectra were pretreated prior to Hard Model fitting by selecting upper and lower wavenumber boundaries as well as a baseline correction as indicated in Tab. 1. The spectral range $395-375 \mathrm{~cm}^{-1}$ is affected by the window material and was hence excluded from the analysis [15].

The initial parameters of the Hard Model are derived from the calculation, i.e. peak positions and relative amplitudes $I_{\text {rel. }}$. Only about five to seven peaks with relative amplitudes of $>1 \%$ of the maximum peak are considered, which is in the typical range of the spectral residuum after the model fit to the spectrum. The peak-shape parameters of Eq. (7) - half-width at half maximum (HWHM) $\gamma$ and the shape parameters (Gaussian Part $\beta$ ) - are set to typical infrared values of $\gamma=5 \mathrm{~cm}^{-1}$ and $\beta$ $=0.7$. Hence, the calculated relative intensities are represented in the peak amplitude only.

In a second step, the most appropriate parameter constraints for the component fit procedure were selected. Since the fundamental wavelength scaling applied to the calculated DFT spectra was observed to partially overcompensate deficiencies of the harmonic wavenumbers of the unscaled spectrum, component shifts (affecting the peak set of a component model as a whole) of up to $15 \mathrm{~cm}^{-}$

${ }^{1}$ were allowed. Additional shifts of individual peaks were allowed to be up to $10 \mathrm{~cm}^{-1}$. In order to account for any type of peak shape, the shape parameter $\beta$ was set free between a purely Gaussian $(\beta=1)$ and a purely Lorentzian shape $(\beta=0)$. HWHM and peak amplitude were allowed to vary by $80 \%$ with respect to the absolute initial value. Both interdepend through the constant area boundary condition.

Thirdly, the parameters to be set free in the fit step had to be selected. We considered three options:

1. Maintaining relative peak intensities (areas) and allowing all peak parameters to change within the boundaries listed in Tab. 2 .

2. Setting free the relative peak intensities, and allowing parameter changes within the boundaries listed in Tab. 2.

3. Setting free all parameters, but using the initial values as starting points for the fit (peak picking).

For comparison with the experiment, the spectral Hard Model was used for peak detection in the measured spectrum. Model peaks of pseudo-Voigt nature were freely fitted to the measured spectra. 
Insignificant peaks obviously representing noise or accounting for asymmetry in a peak were removed from the list, and the remaining significant peaks were again least-squares fitted to the measured spectrum.

In order to include an evaluation of the calculated $\mathrm{T}^{2}$ intensities, only option 1 turned out to be appropriate for our purposes. Option 3 practically gives no difference with a fully free fit to the spectrum, as we applied it for peak identification.

The results of the component fit allow relating $\mathrm{T}^{2}$ intensities obtained from quantum-chemical calculations with the measured peak areas $A_{n}$ through the component weight as a scaling factor. It must, however, be stated that a quantitative correlation between calculated and experimental intensities is not applicable, since the extinction coefficient is an experiment-specific measure and not an absolute one. The spectral parameters can be assessed by comparing their initial value derived from the calculation and their experimental value obtained from the fit.

\section{Results and Discussion}

\subsection{Choice of Density Functional Method}

Preliminary to the detailed discussion on the xylene FIR spectra themselves we want to justify our choice of DFT method which we consider as starting point for future calculations of the less common FIR spectra of liquids [15], polymers [47], and nanoparticles [48] that we measure in our group.

The major criteria for the choice of DFT functional are the differences $\Delta \tilde{v}$ among theoretical gas phase wavenumbers and their experimental counterparts which are usually in considerable agreement. Minor criteria are the relative peak intensities and the structural differences of the considered isomer with different flavors of a given DFT method, i.e. different basis sets or convergence criteria. Contrary to wavenumbers the peak intensities were found to differ among experiment and theory strongly by up to a factor of two, which was somewhat expected, as intensities are difficult to determine accurately either experimentally, as they correlate with the peak area which may be convoluted with neighboring peaks, or with DFT and a triple-zeta basis set $[4,49,50]$.

First of all we selected the DFT functionals BP86, B2PLYP, PWP86, and TPSS0 with different amounts of hybridization that are known to perform well in the calculation of infrared spectra [32]. Calculations were done on a sample basis. In this first round of calculations we compared different methods only and therefore used the TZVP basis set, moderately low convergence criteria, and 
scarcer integration grids, throughout. The obtained wavenumbers were scaled by the fundamental scaling factors given in Sec. 3.1. Comparison with the experimentally obtained wavenumbers showed, that TPSSO on average gave the smallest root mean squares deviations of $9.36 \mathrm{~cm}^{-1}$ closely followed up by BP86 with $11.58 \mathrm{~cm}^{-1}$. Deviations with B2PLYP and PW6B95 were higher with $17.31 \mathrm{~cm}^{-1}$ and $26.81 \mathrm{~cm}^{-1}$, respectively. In all four cases experimental values were systematically underestimated. The relative peak intensities were fully reproduced in the case of PW6B95 only, and to a good extend in the cases of BP86 and TPSSO.

With the second set of benchmark calculations we found that the use of a denser integration grid along with tighter convergence criteria for the SCF and the geometry optimization itself gave identical root mean squares deviations within at most $1 \mathrm{~cm}^{-1}$. The third benchmark was a comparison among the TZVP and the def2-TZVP basis set that resulted in a considerable reduction of the root mean squares deviations by about $5 \mathrm{~cm}^{-1}$ when using def2-TZVP. Hence, the method of choice is D3 corrected TPSSO/def2-TZVP with tight convergence requirements. Its root mean square (RMS) errors are detailed in the following sections with view on the liquid phase (Sec. 4.2.) and scaling (Sec. 4.3.).

\subsection{Calculation of the Liquid Phase}

Since the measurements are not done in the gas phase but rather in the liquid phase, we seek to include solvation into the calculations in the sense that one xylene molecule is embedded in a solution of same isomer xylenes. One possibility would have been through an explicit, but costly, quantum treatment of the surrounding solvent molecules. But for a pure non-polar liquid as any of the xylenes, we consider the continuum solvent model COSMO reliable [45]. RMS errors of wavenumbers with and without COSMO are presented in Tab. 3. The spectra are qualitatively identical and the wavenumbers with and without solvent do not differ much, which can be explained by the xylenes' geometries (cf. Sec. 4.4.) that are very similar in neat liquid and in the gas phase. All bond and dihedral angles are identical and bonds are only slightly elongated by $0.001 \AA$ in the liquid phase.

For $p$-xylene the RMS slightly increases, because the wavenumber of the lowest-intensity peak underestimates the experimental value by nearly $15 \mathrm{~cm}^{-1}$ with the solvation model compared to less than $1 \mathrm{~cm}^{-1}$ in the gas phase. Otherwise, the RMS errors decrease. Therefore we conclude that the solvation model is appropriate for the treatment of liquid xylene. 


\subsection{Scaling Factors}

Scaling factors serve the purpose of bringing the theoretical frequencies obtained from harmonic normal coordinates closer to experimental fundamental frequencies comprising the anharmonicity and phase of a molecule at finite temperature and pressure [21]. For our calculation of FIR spectra we, however, recognized that most FIR wavenumbers get over-corrected by this scaling commonly used for MIR spectra, i.e. that the unscaled frequencies which tend to be slightly too large are systematically underestimated through scaling. We discuss here, why scaling factors should nevertheless be considered for the FIR spectra.

One aspect is that we recognize that our calculated and scaled MIR spectra are in excellent agreement with experimental MIR spectra from the literature $[51,52,53]$. This is not surprising as scaling factors have been optimized for this energy range, i.e. scaling factors do not over-correct. To account for these differences in the performance of scaling factors in the FIR and MIR regions we propose the use non-uniform scaling factors.

Another aspect is that for the FIR region, even with the available scaling factors employed which generally result in the underestimation of individual wavenumbers, the overall RMS errors, shown in Tab. 4, do show a lower average deviation of the theoretical wavenumbers from the experimentally obtained ones.

\subsection{Comparison of Molecular Geometries}

The geometries obtained with D3-TPSSO/def2-TZVP(COSMO) are shown in Fig. 1 with bond distances indicated. Among the different xylene isomers comparable bond lengths (e.g. of aromatic or external C - C bonds) do not differ by more than $0.001 \AA$. Likewise comparable bond angles (e.g. within the aromatic ring) are similar for all isomers. The nearly ideal planarity of the three isomers is determined from one methyl- $C$ over two benzene $C$ to the other methyl- $C$ atom. It sinks from $0.6^{\circ}$ to perfectly planar $0.0^{\circ}$ when going from $o$ - to $p$-xylene, because in $o$-xylene (Fig. 1 (a)) the two methyl groups seek to avoid each other by the two in-plane $\mathrm{C}-\mathrm{H}$ bonds of the methyl groups pointing away from each other leading to a $\mathrm{C}_{2 \mathrm{v}}$ symmetry and by being slightly bent out of the molecular plane. As reported in the literature the $o$-xylene methyl groups are in an inverse orientation, where in-plane C$\mathrm{H}$ bonds point outward in a slightly distorted geometry $[54,55,56,57]$. Contrary to that, in $m$-xylene (Fig. 1 (b)) the nearly in-plane $\mathrm{C}-\mathrm{H}$ bonds of the two methyl groups are oriented in the same direction, leading to a $\mathrm{C}_{\mathrm{S}}$ symmetry, with one pointing slightly up and the other down. Finally, in p-xylene (Fig. 1 
(c)) the two methyl groups take on an anti-orientation in a Newman projection from one methyl-C over the neighboring ring $\mathrm{C}$ to the other such pairs and thus leading to a $\mathrm{C}_{2 \mathrm{~h}}$ molecular symmetry.

\subsection{Discussion of the FIR Xylene Spectra}

\subsection{1. o-Xylene}

In Fig. 2 the experimental FIR extinction coefficient spectrum [15] of $o$-xylene is displayed as blue line in the ranges $510-395 \mathrm{~cm}^{-1}$ and $375-70 \mathrm{~cm}^{-1}$. In the same figure vertical yellow bars represent the theoretical $\mathrm{T}^{2}$ line spectrum and a red line its Hard Model fit $S$ described in Sec. 3.2. The peak positions $\tilde{v}$, their shifts $\Delta \tilde{v}$ with the Hard Model fit, and relative intensities $\mathrm{I}_{\text {rel }}=\mathrm{T}^{2} / \mathrm{T}^{2}$ max for theory and $I_{\text {rel }}=A / A_{\max }$ for experiment are listed in Tab. 5 for the three data sets and an experimental reference [2]. Moreover, a description of the vibration is given which is likewise represented as visualization in Fig. 2. Further we assigned the symmetries of the $\mathrm{C}_{2 \mathrm{v}}$ point group that apply to each vibration to compare with the assignment of Green, Kynaston and Gebbie [2].

For $o$-xylene the DFT calculation reveals seven peaks with non-zero $T^{2}$ values. Six of them are used in the Hard Model fit (cf. Tab. 5) excluding the range of $375-395 \mathrm{~cm}^{-1}$ as the experimental spectrum is dominated here by background signals. Four of them can be seen in our experimental data, five have been reported in an older experiment from the 1960s [2].

The two strongest peaks at about $255(255) \mathrm{cm}^{-1}$ and 435 (433) $\mathrm{cm}^{-1}$ in our [15] (the older [2]) experimental data have their counterparts in the calculated spectrum at the moderately deviating wavenumbers of $248 \mathrm{~cm}^{-1}$ and $430 \mathrm{~cm}^{-1}$ and with the same intensity ordering. By inspecting the animations of the calculated vibrations shown in Fig. 2 we assign the first one to a wagging motion of both methyl groups, which results in $b_{1}$ symmetry. This is in contrast to an assignment of $a_{1,2}$ symmetry reported in the earlier publication [2], however their symmetry assignment was done when no animation was available. The second mode appearing at higher wavenumbers is in agreement with the older assignment and corresponds to $a b_{1}$ symmetric bending motion of methyl groups. Here, both carbon atoms neighboring the methyl groups are bent out of the ring plane with the effect of both methyl groups being bent towards the aromatic ring in a symmetric fashion. Both motions go along with an oscillation of the molecule's dipole moment out of the molecular plane which is the largest among all discussed vibrations.

A pair of peaks having comparably lower intensities appears at theoretical frequencies $390 \mathrm{~cm}^{-1}$ and $288 \mathrm{~cm}^{-1}$ which comprises rocking $\left(b_{2}\right)$ and scissoring $\left(a_{1}\right)$ motions of both methyl groups. Here the 
dipole moment only changes within the molecular plane which accounts for the low intensity. In addition, the contributing background signals complicate the peak assignment leading to the disagreement between the theoretical peak at $288 \mathrm{~cm}^{-1}$, our experimental and the older experimental data by about 9 and $37 \mathrm{~cm}^{-1}$, respectively. For the case of the $390 \mathrm{~cm}^{-1}$ peak the difference to the two experiments is equal, $14-15 \mathrm{~cm}^{-1}$, although in the older experiment the symmetry was assigned differently.

Another group of peaks appears in the Hard Model fit (theory) spectrum at $110(104) \mathrm{cm}^{-1}$ and 144 (151) $\mathrm{cm}^{-1}$ and corresponds to the same- and inverse-direction torsion motion of the two methyl groups having $a_{2}$ and $b_{1}$ symmetry, respectively. The latter is symmetry allowed, indicates a moderate change of dipole moment and was hence found in two older experiments [2,58], however with deviating wavenumber of $180 \mathrm{~cm}^{-1}$. There a symmetry assignment was not made. Note at this point that the discussed torsion peaks might in cases be infrared active only in liquid but not in gas phase spectra. This would be caused by neighboring molecules hindering the free methyl rotation thus leading to a significant increase of the rotational barrier compared to the one found in the gas phase $[59,60]$. Here we confirm what was found by others [58]: in $o$-xylene the hindered rotation is a local effect of the isolated molecule, because the torsional peak is also present in the qualitatively similar gas phase spectrum from our calculation (the experiment [58]) at $153 \mathrm{~cm}^{-1}\left(162 \pm 5 \mathrm{~cm}^{-1}\right)$. Moreover, our gas phase calculations of several $o$-xylene conformers reveal that the barrier height is about ten times larger than the vibrational energy.

At the lowest energy range recorded distortions of the carbon skeleton start to appear as the $b_{2}$ ring stretching motion, which is in the Hard Model fit (theoretically) at low (medium) intensity and at position $509(487) \mathrm{cm}^{-1}$.

\subsection{2. $m$-Xylene}

The spectra of $m$-xylene are displayed in Fig. 3 in the ranges $547-395 \mathrm{~cm}^{-1}$ and $375-70 \mathrm{~cm}^{-1}$. The color coding is the same as in Sec. 4.5.1., and spectral parameters are summarized in Tab. 6. The calculated $m$-xylene spectrum has a total number of ten peaks and experimentally eight of these can be resolved. It resembles the $o$-xylene spectrum in most respects which is originating from the fact that both isomers have the same type of vibrations that are all symmetry allowed in the $C_{s}$ symmetry of $m$-xylene. One of the highest-intensity peaks is again corresponding to the bending motion of the aromatic ring where both carbon atoms neighboring the methyl groups move to the same direction out of the molecular plane at $432(436) \mathrm{cm}^{-1}$ in the Hard Model fit and in experiment (DFT 
calculation). Note that the intensity ordering of calculated and experimental bands is deviating from each other here, so that the peak is only second highest in the experiment. Despite its high intensity and very similar position as in o-xylene it had not been detected in the 1960s [2].

The most intense experimental peak with second-highest intensity in the calculation, appearing at 204 (195) $\mathrm{cm}^{-1}$ is of a" symmetry and corresponds to a wagging vibration like in o-xylene, i.e. a concerted motion of the two methyl groups out of the molecular plane to the same side. Our wavenumber and intensity are in good agreement with earlier works, except that the authors there assigned $\mathrm{a}_{1}$ symmetry to the peak assuming another than the $\mathrm{C}_{\mathrm{S}}$ point group [2].

The counterpart to this out-of-plane vibration with both methyl groups moving to opposite directions (twisting motion) also has a" symmetry and is detected in the DFT calculation at $231 \mathrm{~cm}^{-1}$ and with very low intensity due to a lower change of dipole moment. In the Hard Model fit it is assigned in the experimental spectrum to $226 \mathrm{~cm}^{-1}$.

Again, two low-intensity peaks at similar frequency as in o-xylene can be assigned to a' in-plane bending motions of the two methyl groups either to the opposite (scissoring) or the same (rocking) direction. The former appears in the experiment (theory) at $277(260) \mathrm{cm}^{-1}$. The assignment is complica ted for such low-intensity peaks and deviations are about $17 \mathrm{~cm}^{-1}$. But the old and the new experimental data are in perfect agreement including the mirror plane symmetry of the vibration. The peak of the latter is detected at a theoretical wavenumber of $376 \mathrm{~cm}^{-1}$ which is experimentally overlaid by noise. It deviates by about $26 \mathrm{~cm}^{-1}$ from our recent and the old experimental value [2].

A pair of low-intensity methyl group torsion peaks appears at $85(86) \mathrm{cm}^{-1}$ and $133(120) \mathrm{cm}^{-1}$ in the Hard Model fit (theory) that had not been reported before. In contrast to $o$-xylene, here either of the methyl groups is undergoing torsional motion while the other is not moving. Both are non-symmetric vibrations. However, both a torsion of the two methyl groups into opposite or the same direction would be symmetry allowed for $C_{s} m$-xylene (both a"). The combination of these symmetric torsions causes the appearance of the observed two peaks at similar intensity. Due to the above symmetry and combination considerations we assign $\left\{a^{\prime \prime}\right\}$ in curly brackets.

Finally a number of high-wavenumber peaks belong to the spectrum that originate from vibrations of the aromatic ring skeleton of $a^{\prime}, a^{\prime \prime}$, and a' symmetry. The fit of the spectral Hard Model based on the theoretical wavenumbers and intensities is challenging in this range. The three theoretical peaks, one at $497 \mathrm{~cm}^{-1}$ with high and two at 511 and $521 \mathrm{~cm}^{-1}$ with significantly lower intensity do not properly match the peaks identified in the experiment at 489, 513 and $535 \mathrm{~cm}^{-1}$ with very similar intensities. The fit of the Hard Model to the spectrum under the constraint of fixed relative peak intensities 
(option 1 in Sec. 3.2) results in peaks at $511 \mathrm{~cm}^{-1}$ (high intensity) and 528 and $537 \mathrm{~cm}^{-1}$ (low intensity), which is indeed minimizing the spectral residuals in this range, but which cannot properly explain the experimental peaks.

\subsection{3. $p$-Xylene}

The $p$-xylene spectra in the ranges $550-395 \mathrm{~cm}^{-1}$ and $375-50 \mathrm{~cm}^{-1}$ are found in Fig. 4 using the same color coding as previously described (Secs. 4.5.1. and 4.5.2.). The corresponding spectral parameters are collected in Tab. 7.

$P$-xylene has considerably different spectroscopic properties than $o$ - and $m$-xylene but also it has different $C_{2 h}$ symmetry. The number of only two infrared-allowed IRREPs leads to an overall decreased number of peaks. Fitting was done for a total number of five theoretically predicted peaks, three of which are found experimentally with a significant intensity. Only a single lower-intensity in-plane bending motion of $a_{u}$ symmetry is present in the experiment (calculation) at $292(278) \mathrm{cm}^{-1}$ with a barely changing dipole moment. Moreover, there is only a single $b_{u}$ out-of-plane bending motion which appears in the theoretical spectrum at $78 \mathrm{~cm}^{-1}$. It has admixes of an $\mathrm{a}_{\mathrm{u}}$-symmetric torsion of both methyl groups into the same direction. Another peak that belongs purely to that torsional motion appears at $149(160) \mathrm{cm}^{-1}$. It was the only peak reported in the earlier experiment at $166 \mathrm{~cm}^{-1}$ [2] with $b_{3 \mathrm{u}}$ symmetry, based on the $D_{2 h}$ symmetry of the carbon skeleton only and having very strong intensity, whereas our findings suggest it not to be intense. The methyl-group torsion into the inverse direction does not appear in the spectrum as it does not change the dipole moment of $p$-xylene $\left(b_{g}\right.$ symmetry).

Only for the case of bending motion of the aromatic ring we find more peaks in $p$-xylene than for other isomers. One of these is an $\mathrm{a}_{\mathrm{u}}$-symmetric bending motion of $\mathrm{C}-\mathrm{H}$ bonds against the four nonmethylated C-atoms of the aromatic ring that was previously not observed. It is calculated to be at $404 \mathrm{~cm}^{-1}$ and is weak, so that experimental detection at the given wavenumber was impossible, the Hard Model fits the peak to $412 \mathrm{~cm}^{-1}$. The other strong peak found at $484\left(472 \mathrm{~cm}^{-1}\right)$ corresponds to the bending motion of the same four carbon atoms all to the same side of the molecular plane with $b_{u}$ symmetry. There is no reference for this peak, because it lies beyond the detection range of the 1960s experiment. 


\subsection{Global Trends in the Row of Xylenes}

A global view across the three xylene isomers helps to further understand for which of the vibrations the theoretical approach and the measurement differ most. With further comparison of the xylene FIR spectra a few generalities appear. Due to the same $C_{2 v}$ symmetry of the carbon skeleton only, the spectra of $o$ - and $m$-xylene (total $m$-xylene has $C_{s}$ symmetry) are composed of basically the same vibrations at slightly shifted energies, whereas $p$-xylene with other mirror planes in its $C_{2 h}$ molecular symmetry has a noticeably different spectrum comprising fewer peaks. Nonetheless, all these isomers share a unique pattern of characteristic vibrations at typical group frequencies.

The two lowest frequency vibrations appear below $170 \mathrm{~cm}^{-1}$ in the calculated spectra (liquid and gas phase) and one of them also in the experimental one. They are torsions of the two methyl groups with slightly varying pattern. Focusing on the agreement among theoretical prediction and measured spectrum we observe by the Hard Model fit that peak positions differ on average by $\Delta \tilde{v}=7.2 \mathrm{~cm}^{-1}$. We want to state here that discrepancies between calculated and experimental peaks are due to the relatively low intensities of the methyl-torsion peaks, which make particularly the assignment of the experimental peaks with the Hard Model fit in presence of the spectral background ambiguous. The largest deviation is $\Delta \tilde{v}=12.4 \mathrm{~cm}^{-1}$ for the higher wavenumber peak of $m$-xylene. In contrast, the $p$ xylene peaks differ least, particularly the low energy peak at $77 \mathrm{~cm}^{-1}$ of an overlay of a methyl-group torsion and a wagging motion.

The peaks in the range of $230-250 \mathrm{~cm}^{-1}$ correspond to out-of-plane bending motions of the two methyl groups, which are completely symmetry forbidden in $p$-xylene. In the $C_{2 v}$ symmetry the twisting vibration is not symmetry allowed, and in the $C_{S}$ symmetry the related peak appears with very low intensity. In contrast, the wagging peak is the most intense one in the experimental spectra of $o$ - and $m$-xylene, but not in the calculation of $p$-xylene. Their wavenumbers are theoretically moderately underestimated by about $\Delta \tilde{v}=7.3 \mathrm{~cm}^{-1}$ for $o$-xylene and by $\Delta \tilde{v}=8.6 \mathrm{~cm}^{-1}$ for the misomer. We connect the trend for underestimation to the empirical frequency shift, which we find to over-correct theoretically calculated wavenumbers.

Two low-intensity in-plane bending motions of both methyl groups follow in the wavenumber range of $250-420 \mathrm{~cm}^{-1}$. Only in the case of $p$-xylene one of them is symmetry forbidden. Due to the very low intensities of these peaks assignment is typically complicated and leads to deviations in the order of $\Delta \tilde{v}=10 \mathrm{~cm}^{-1}$. This happens particularly in the high-wavenumber range around $400 \mathrm{~cm}^{-1}$ of the experimental data used due to the experimental noise. 
In the detected spectral range $\tilde{v}>400 \mathrm{~cm}^{-1}$ one or two bending motions of the aromatic ring in the molecular plane appear. Experimental and theoretical peak positions are in good agreement with deviations of about $5-10 \mathrm{~cm}^{-1}$ for all xylenes. On the one hand this goes back to the facilitated assignment of this first- or second-highest intensity peak, for which the relative intensities in theory and experiment agree well ( $o$ - and $p$-isomer). On the other hand the scaling factors (Sec. 4.3.) are well-suited in this range of higher wavenumbers. Lower-intensity ring stretches are assigned less accurately.

In conclusion, in all xylene spectra we find the same characteristic vibrations appear at similar wavenumbers and intensities. Experimental intensity ordering is occasionally not reproduced by the theory, but wavenumbers are typically reproduced within about $10 \mathrm{~cm}^{-1}$.

\section{Conclusion}

Far-infrared spectra of neat liquids of the three xylene isomers recorded by ellipsometry in attenuated total reflection mode were analyzed by means of density functional theory calculations. As the FIR range is much less well investigated than the MIR range, the critical focus lies on the applicability of computational concepts typically used as a standard in calculations of MIR spectra. The choice of functionals and basis sets, the treatment of solvent effects through polarizable continuum models, and the account for anharmonicity through scaling factors were evaluated. For better visual comparisons with experimental spectral data beyond the commonly used uniform line broadening we have developed a new analysis procedure based on spectral Hard Modeling. Its core piece is the fit of calculated line spectra to experimental FIR spectra with a quantitative track record on the modifications. With the DFT and Hard Modeling protocol developed we are able to reproduce and explain the experimental spectra and discuss trends among the three isomers of xylene.

\section{Acknowledgments}

I.B. and A.B. thank M. Pernpointner from Heidelberg University for supporting the external bachelor thesis of I.B. at Helmholtz-Zentrum Berlin. We further thank our HZB colleagues K. Atak, T. Brandenburg, and M. Fromme for sharing their experience in running ORCA calculations. A. B. acknowledges funding of her position through by the Volkswagen foundation [Freigeist fellowship no. 89525]. This research itself did not receive any specific grant from funding agencies in the public, commercial, or non-for-profit sectors. 
[1] M. Derrick, D. Stulik, and J. M. Landry, Infrared Spectroscopy in Conservation Science, The Getty Conservation Institute, 1999.

[2] J. H. Green, W. Kynaston, and H. A. Gebbie, "Far Infra-red Spectroscopy of Benzene Derivatives by Interferometry," Nature, vol. 159, p. 595 - 596, 1962.

[3] H. H. Mantsch and D. Naumann, "Terahertz spectroscopy: The renaissance of far infrared spectroscopy," J. Mol. Struct., vol. 964, pp. 1 - 4, 2010.

[4] F. L. Gervasio, G. Cardini, P. R. Salvi, and V. Schettino, "Low-Frequency Vibrations of all-transRetinal: Far-Infrared and Raman Spectra and Density Functional Calculations," J. Phys. Chem. A, vol. 102, pp. 2131 - 2136, 1998.

[5] B. Fischer, M. Hoffmann, H. Helm, G. Modjesch, and P. U. Jepsen, "Chemical recognition in terahertz time-domain spectroscopy and imaging," Semicond. Sci. Tech., vol. 20, pp. S246S253, 2005.

[6] I. Jones, T. J. Rainsford, S. P. Mickan, and D. Abbott, "Ab initio molecular orbital theory: a tool for THz spectroscopic investigation," Proc. of SPIE, Vol. 6038, pp. 60381I-1 - 60381I-11, 2006.

[7] H. Ciurla, J. Michalski, J. Hanuza, M. Mączka, T. Talik, and Z. Talik, "Molecular structure, IR and Raman spectra as well as DFT chemical calculations for alkylaminoacetylureas: vibrational characteristics of dicarbonylimide bridge," Spectrochim. Acta A, vol. 64, pp. 34 - 46, 2006.

[8] P. U. Jepsen, and S. J. Clark, "Precise ab-initio prediction of terahertz vibrational modes in crystalline systems," Chem. Phys. Lett., vol. 442, pp. 275 - 280, 2007.

[9] M. Heng, S. De-Heng, H. Jun, and P. Yu-Feng, "Simulation study on terahertz vibrational absorption in liquid crystal compounds," Chinese Phys. B, vol. 18, pp. 1084 - 1088, 2008.

[10] Q. Song, Y. Zhao, R. Zhang, X. Liu, L. Dong, and W. Xu, "Measurement and DFT Calculation on Terahertz Spectroscopy of 4-aminobenzoic Acid," J. Infrared Millim. Te.," vol. 31, pp. 310-318, 2010.

[11] K. Itoh, A. Ikeda, T. Iwamoto, and S. Nishizawa, "DFT calculation analysis of terahertz timedomain spectra of polyalanines," J. Mol. Struct., vol. 1006, pp. 52 - 58, 2011.

[12] D. Marx and J. Hutter, "Ab initio molecular dynamics: Theory and Implementation" in Modern Methods and Algorithms of Quantum Chemistry, Jülich: John von Neumann Institute for Computing, vol. 1, pp. 301 - 449, 2000.

[13] H.-D. Meyer, F. Gatti, and G. A. Worth, Eds., Multidimensional Quantum Dynamics, Weinheim: Wiley-VCH, 2009

[14] M. A. Suhm and R. O. Watts, "Quantum Monte Carlo Studies of Vibrational States in Molecules and Clusters", Phys. Rep., vol. 204, pp. 293 - 329, 1991.

[15] J. Beckmann, B. Marchetti, L. S. von Chrzanowski, E. Ritter, L. Puskar, E. F. Aziz, and U. Schade "Optical Constants of Harmful and Highly Energetic Liquids for Application to THz Screening Systems," IEEE Trans. THz Sci. Technol., vol. 6, pp. 396 - 407, 2016.

[16] F. Alsmeyer, H.-J. Koß, and W. Marquardt, "Indirect Spectral Hard Modeling for the Analysis of Reactive and Interacting Mixtures," Appl. Spectros., vol. 58, pp. 957 - 985, 2004.

[17] J. F. Federici, B. Schulkin, F. Huang, D. Gary, R. Barat, F. Oliveira, and D. Zimdars, "THz imaging and sensing for security applications - explosives, weapons and drugs," Semicond. Sci. Technol., vol. 20, pp. S266 - S280, 2005.

[18] N. Krumbholz, C. Jansen, M. Scheller, T. Müller-Wirts, S. Lübbecke, R. Holzwarth, R. 
Scheunemann, R. Wilk, B. Sartorius, H. Roehle, D. Stanze, J. Beckmann, L. S. von Chrzanowski, U. Ewert, and M. Koch, "Handheld Terahertz Spectrometer for the Detection of Liquid Explosives," Proc. SPIE, vol. 7485, p. 748504, 2009.

[19] W. Demtröder, Atoms, Molecules and Photons - An Introduction to Atomic-, Molecular- and Quantum Physics, $2^{\text {nd }}$ Edition, Springer Berlin, Heidelberg, 2006, 2010.

[20] J. M. Hollas, Modern Spectroscopy, $4^{\text {th }}$ Edition, John Wiley \& Sons Ltd, The Atrium, Southern Gate, Chichester, West Sussex PO19 8SQ, England, 2004.

[21] A. P. Scott and L. Radom "Harmonic Vibrational Frequencies: An Evaluation of Hartree-Fock, Møller-Plesset, Quadratic Configuration Interaction, Density Functional Theory, and Semiempirical Scale Factors," J. Phys. Chem., vol. 100, pp. 16502 - 16513, 1996.

[22] F. Alsmeyer and W. Marquardt, "Automatic Generation of Peak-Shaped Models," Appl. Spectros., vol. 58, pp. 986 - 994, 2004.

[23] E. Kriesten, F. Alsmeyer, A. Bardow, and W. Marquardt, "Fully automated indirect hard modeling of mixture spectra," Chemometr. Intell. Lab., vol. 91, pp. 181 - 193, 2008.

[24] F. Neese, "The ORCA program system," WIREs Comput. Mol. Sci., vol. 2, pp. 73 - 78, 2012.

[25] A. D. Becke, "Density-functional exchange energy approximation with correct asymptotic behaviour," Phys. Rev. B, vol. 38, pp. 3098 - 3100, 1988.

[26] J. P. Perdew, "Density-functional approximation for the correlation energy of the inhomogeneous electron gas," Phys. Rev. B, vol. 33, pp. 8822 - 8824, 1986.

[27] J. P. Perdew, "Erratum: Density-functional approximation for the correlation energy of the inhomogeneous electron gas," Phys. Rev. B, vol. 34, p. 7406, 1986.

[28] Y. Zhao and D. G. Truhlar, "Design of Density Functionals That Are Broadly Accurate for Thermochemistry, Thermochemical Kinetics, and Nonbonded Interactions," J. Phys. Chem. A, vol. 109, pp. 5656 - 5667, 2005.

[29] S. Grimme, "Accurate Calculation of the Heats of Formation for Large Main Group Compounds with Spin-Component Scaled MP2 Methods," J. Phys. Chem. A, vol. 109, pp. 3067 - 3077, 2005.

[30] S. Grimme, "Semiempirical hybrid density functional with perturbative second-order correlation," J. Chem. Phys., vol. 124, p. 034108, 2006.

[31] L. Goerigk and S. Grimme, "A thorough benchmark of density functional methods for general main group thermochemistry, kinetics, and noncovalent interactions," Phys. Chem. Chem. Phys. vol. 13, pp. 6670 - 6688, 2011.

[32] M. K. Kesharwani, B. Brauer, and J. M. L. Martin, "Frequency and Zero-Point Vibrational Energy Scale Factors for Double-Hybrid Density Functionals (and Other Selected Methods): Can Anharmonic Force Fields Be Avoided?," J. Phys. Chem. A, vol. 119, pp. 1701 - 1714, 2015.

[33] A. Schäfer, H. Horn, and R. Ahlrichs, "Fully Optimized Contracted Gaussian Basis Sets for Atoms Li to Kr," J. Chem. Phys., vol. 97, pp. 2571 - 2577, 1992.

[34] F. Weigend and R. Ahlrichs, "Balanced basis sets of split valence, triple zeta valence and 
quadruple zeta valence quality for $\mathrm{H}$ to Rn: Design and assessment of accuracy," Phys. Chem. Chem. Phys., vol. 7, pp. 3297 - 3305, 2005.

[35] E. J. Baerends, D. E. Ellis, and P. Ros, "Self-consistent molecular Hartree-Fock-Slater calculations I. The computational procedure," Chem. Phys., vol. 2, pp. 41 - 51, 1973.

[36] J. L. Whitten, "Coulombic potential energy integrals and approximations," J. Chem. Phys., vol. 58, pp. 4496 - 4501, 1973.

[37] B. I. Dunlap, J. W. D. Connolly, and J. R. Sabin, "On some approximations in applications of Xa theory," J. Chem. Phys., vol. 71, pp. 3396 - 3402, 1979.

[38] C. Van Alsenoy, "Ab initio calculations on large molecules: The multiplicative integral approximation," J. Comput. Chem., vol. 9, pp. 620 - 626, 1988.

[39] F. Weigend and M. Häser, "RI-MP2: First Derivatives and Global Consistency," Theor. Chem. Acc., vol. 97, pp. 331 - 340, 1997.

[40] F. Neese, F. Wennmohs, A. Hansen, and U. Becker, "Efficient, approximate and parallel Hartree-Fock and hybrid DFT calculations. A 'chain-of-spheres' algorithm for the Hartree-Fock exchange," Chem. Phys., vol. 356, pp. 98 - 109, 2009.

[41] R. Iszák and F. Neese, "An overlap fitted chain of spheres exchange method," J. Chem. Phys., vol. 135, p. 144105, 2011.

[42] T. Pentrenko, S. Kossmann, and F. Neese, "Efficient time-dependent density functional theory approximations for hybrid density functionals: Analytical gradients and parallelization," $J$. Chem. Phys., vol. 134, p. 054116, 2011.

[43] S. Grimme, J. Antony, S. Ehrlich, and H. Krieg, "A consistent and accurate ab initio parametrization of density functional dispersion correction (DFT-D) for the 94 elements $\mathrm{H}-\mathrm{Pu}$," J. Chem. Phys., vol. 132, p. 154104, 2010.

[44] S. Grimme, S. Ehrlich, and L. Goerigk, "Effect of the damping function in dispersion corrected density functional theory," J. Comp. Chem., vol. 32, pp. 1456 - 1465, 2011.

[45] S. Sinnecker, R. Arivazhagan, A. Klamt, M. Diedenhofen, and F. Neese, "Calculation of Solvent Shifts on Electronic g-Tensors with the Conductor-Like Screening Model (COSMO) and Its SelfConsistent Generalization to Real Solvents (Direct COSMO-RS)," J. Phys. Chem. A, vol. 110, pp. 2235 - 2245, 2006.

[46] I. M. Alecu, J. Zheng, Y. Zhao, and D. G. Truhlar, "Computational Thermochemistry: Scale Factor Databases and Scale Factors for Vibrational Frequencies Obtained from Electronic Model Chemistries," J. Chem. Theory Comput., vol. 6, pp. 2876 - 2887, 2010.

[47] A. D. O. Bawagan, S. J. Hamrock, M. Schaberg, I. Yousef, E. Ritter and U. Schade, "Far-infrared studies on Nafion and perfluoroimide acid (PFIA) and their alkali salts," Vib. Spectrosc., vol. 75, p. $213-217,2014$.

[48] W. Kuehn, K. Reimann, M. Woerner, T. Elsaesser, R. Hey, and U. Schade, "Strong Correlation of Electronic and Lattice Excitations in GaAs/AIGaAs Semiconductor Quantum Wells Revealed by Two-Dimensional Terahertz Spectroscopy," Phys. Rev. Lett., vol. 107, pp. 067401-1 - 067401-5, 2011. 
[49] C. Willmer and M. Fricker, Density Functional Theory of Molecules, Clusters, and Solids, Chapman and Hall, 1996.

[50] V. Barone (Ed.), Computational Strategies for Spectroscopy: from Small Molecules to Nano Systems, John Wiley \& Sons Inc., Hoboten, New Jersey, 2012.

[51] NIST Chemistry WebBook. Available at: http://webbook.nist.gov/cgi/cbook.cgi? ID=C95476\&Units=SI\&Type=IR-SPEC\&Index=2\#IR-SPEC (accessed on September 7, 2016)

[52] NIST Chemistry WebBook. Available at: http://webbook.nist.gov/cgi/cbook.cgi? $\underline{I D}=C 108383 \&$ Units=SI\&Type=IR-SPEC\&Index=3\#IR-SPEC (accessed on September 7, 2016)

[53] NIST Chemistry WebBook. Available at: http://webbook.nist.gov/cgi/cbook.cgi? ID=C106423\&Units=SI\&Type=IR-SPEC\&Index=3\#IR-SPEC (accessed on September 7, 2016)

[54] W. R. Woolfenden and D. M. Grant "Carbon-13 Magnetic Resonance. V.1 Conformational Dependence of the Chemical Shifts in the Methylbenzenes," J. Am. Chem. Soc., vol. 88, pp. 1496 - 1502, 1966.

[55] K. M. Gough and B. R. Henry "Gas-phase overtone spectral investigation of inequivalent aryl and alkyl carbon-hydrogen (C-H) bonds in toluene and the xylenes," J. Phys. Chem., vol. 88, pp. 1298 - 1302, 1984.

[56] R. Disselkamp, E. R. Bernstein, J. I. Seeman, and H. V. Secor "Minimum energy conformation of ortho-xylene in its ground and first excited electronic states," J. Chem. Phys., vol. 97, pp. 8130 - 8136, 1992.

[57] J. I. Seeman, H. V. Secor, R. Disselkamp, and E. R. Bernstein "Conformational analysis through selective isotopic substitution: supersonic jet spectroscopic determination of the minimum energy conformation of o-xylene," J. Chem. Soc., Chem. Commun., vol. 95, pp. 713 - 714, 1992.

[58] G. W. F. Pardoe, S. J. Larson, H. A. Gebbie, S. J. Strickler, K. C. Ingham, and D. G. Johnson “FarInfrared Spectrum of ortho-Xylene," J. Chem. Phys., vol. 52, pp. 6426-6427, 1970.

[59] G. A. Guirgis, S. Bell, P. Groner, C. Zheng, and J. R. Durig "Infrared, Raman and far infrared spectra, ab initio calculations, and internal rotation of 3-fluoro-3-methyl-1-butyne," Phys. Chem. Chem. Phys., vol. 6, pp. 3919 - 3927, 2004.

[60] D. T. Durig, S. Shen, Y. Li, and J. R. Durig "Conformational stabilities and structural parameters of (CH3)nCH3-n CFO molecules," Spectrochim. Acta A, vol. 60, pp. 1481-1504, 2004. 


\section{Figure Captions}

Figure 1: Geometries of o-xylene (a), m-xylene (b), and p-xylene (c) in the liquid phase obtained with TPSSO/def2-TZVP, tight convergence criteria, and usage of molecular symmetry as described in the text. The bond lengths are given in $\AA$.

Figure 2: Stacked plot of the experimental FIR extinction coefficient spectrum of o-xylene (bottom,

blue, k), theoretically calculated line spectrum (middle, yellow, $T^{2}$ ), and Hard Model fit of the theoretical peaks onto the spectrum (top, red, S). Note that the theoretical intensities are $T^{2}$ values in $\mathrm{km} / \mathrm{mol}$ rescaled by a factor of 0.01 to be on the same order of magnitude as the extinction coefficients. For each peak the corresponding vibration is indicated qualitatively.

Figure 3: Stacked plot of the experimental FIR extinction coefficient spectrum of m-xylene (bottom, blue, k), theoretically calculated line spectrum (middle, yellow, $T^{2}$ ), and Hard Model fit of the theoretical peaks onto the spectrum (top, red, S). Note that the theoretical intensities are $T^{2}$ values in $\mathrm{km} / \mathrm{mol}$ rescaled by a factor of 0.01 to be on the same order of magnitude as the extinction coefficients. For each peak the corresponding vibration is indicated qualitatively.

Figure 4: Stacked plot of the experimental FIR extinction coefficient spectrum of p-xylene (bottom, blue, k), theoretically calculated line spectrum (middle, yellow, $T^{2}$ ), and Hard Model fit of the theoretical peaks onto the spectrum (top, red, S). Note that the theoretical intensities are $T^{2}$ values in $\mathrm{km} / \mathrm{mol}$ rescaled by a factor of 0.01 to be on the same order of magnitude as the extinction coefficients. For each peak the corresponding vibration is indicated qualitatively. 
Table 1: Pretreatment of experimental spectra.

\begin{tabular}{ccc}
\hline isomer & Fitting regions $\left(\mathrm{cm}^{-1}\right)$ & Baseline correction \\
\hline$o$-xylene & $(510-395)+(375-70)$ & Rubber band subtraction \\
$m$-xylene & $(547-395)+(375-70)$ & Straight line subtraction \\
$p$-xylene & $(550-395)+(375-50)$ & Rubber band subtraction \\
\hline
\end{tabular}


Table 2: Fit constraint settings for spectral Hard Model fit (Eq. (6)).

\begin{tabular}{|c|c|c|c|c|}
\hline Parameter name & Symbol & Initial value & Constraint & Coupling of constraints \\
\hline Component shift & & $0 \mathrm{~cm}^{-1}$ & $\pm 15 \mathrm{~cm}^{-1}$ & Set free. \\
\hline Peak position & $\omega$ & Theory $(\omega)$ & $\pm 10 \mathrm{~cm}^{-1}$ & Set free. \\
\hline Peak amplitude & $\alpha$ & Theory $\left(T^{2}\right)$ & $\pm 80 \%$ & Coupled to HWHM. \\
\hline HWHM & $\gamma$ & $5 \mathrm{~cm}^{-1}$ & $\pm 80 \%$ & Coupled to amplitude. \\
\hline Gaussian part & $\beta$ & 0.7 & $0 \ldots 1$ & Set free. \\
\hline Peak area & & Calculated from $\mathrm{T}^{2}$ & & Relative areas kept constant. \\
\hline $\begin{array}{l}\text { Component } \\
\text { weight }\end{array}$ & $w_{i}$ & \multicolumn{3}{|c|}{ Used as a scaling factor between $\mathrm{T}^{2}$ and measured spectrum. } \\
\hline
\end{tabular}


Table 3: RMS errors of scaled wavenumbers $\tilde{v}$ in gas and liquid phase given in $\mathrm{cm}^{-1}$.

\begin{tabular}{lcc}
\hline & gas phase & liquid phase \\
\hline o-xylene & 7.42 & 6.42 \\
$m$-xylene & 15.05 & 10.63 \\
$p$-xylene & 10.12 & 11.18 \\
\hline
\end{tabular}


Table 4: RMS errors of scaled and unscaled wavenumbers $\tilde{v}$ given in $\mathrm{cm}^{-1}$.

\begin{tabular}{lcc}
\hline & scaled & unscaled \\
\hline o-xylene & 6.42 & 12.67 \\
$m$-xylene & 10.63 & 12.75 \\
$p$-xylene & 11.18 & 16.09 \\
\hline
\end{tabular}


Table 5: Theoretical (Theo), Hard Model fit (HM), and present (Expt) [15] and reference (Ref) [2] experimental peak positions $\tilde{v}$ in $\left(\mathrm{cm}^{-1}\right)$, relative intensities $I_{\text {rel }}=T^{2} / T_{\text {max }}^{2}=A / A_{\max }$ wavenumber shifts $\Delta \tilde{v}$, and corresponding vibrations of o-xylene in $C_{2 v}$ symmetry. The reference intensities abbreviate with $v=v e r y, s=s t r o n g, m=m e d i u m$, and $w=$ weak. Symmetries of the vibrations have been assigned with Schoenfließ symbols.

\begin{tabular}{ccccccccl}
\hline$\tilde{v}^{\text {Theo }}$ & $\mathrm{I}_{\text {rel }}^{\text {Theo }}$ & $\tilde{v}^{\text {HM }}$ & $\Delta \tilde{v}^{\text {HM }}$ & $\tilde{v}^{\text {Expt }}$ & $\mathrm{I}_{\text {rel }}^{\text {Expt }}$ & $\tilde{v}^{\text {Ref }}$ & $\mathrm{I}_{\text {rel }}^{\text {Ref }}$ & Vibration \\
\hline 103.7 & 0.01 & 109.4 & 5.7 & 85.4 & 0.08 & - & - & sym Me torsion, $\mathrm{a}_{2}$ \\
150.8 & 0.08 & 143.8 & -7.0 & - & - & 180 & $\mathrm{w}$ & anti Me torsion, $\mathrm{b}_{1}$ \\
247.6 & 1.00 & 254.9 & 7.3 & 254.9 & 1.00 & 255 & $\mathrm{vs}-\mathrm{a}_{1,2}$ & Me wagging, $\mathrm{b}_{1}$ \\
288.1 & 0.04 & 279.5 & -8.6 & - & - & 325 & $\mathrm{w}$ & Me scissoring, $\mathrm{a}_{1}$ \\
390.3 & 0.06 & - & - & 405.3 & 0.06 & 404 & $\mathrm{~m}-\mathrm{b}_{1}$ & Me rocking, $\mathrm{b}_{2}$ \\
429.8 & 0.64 & 435.2 & 5.4 & 435.1 & 0.51 & 433 & $\mathrm{~s}-\mathrm{b}_{1}$ & ring bending, $\mathrm{b}_{1}$ \\
487.4 & 0.13 & 508.7 & 21.3 & - & - & - & - & ring stretch, $\mathrm{b}_{2}$ \\
\hline
\end{tabular}


Table 6: Theoretical (Theo), Hard Model fit (HM), and present (Expt) [15] and reference (Ref) [2] experimental peak positions $\tilde{v}$ in $\left(\mathrm{cm}^{-1}\right)$, relative intensities $I_{\text {rel }}=T^{2} / T_{\text {max }}^{2}=A / A_{\max }$ wavenumber shifts $\Delta \tilde{v}$, and corresponding vibrations of $m$-xylene in $C_{s}$ symmetry. The reference intensities abbreviate with $v=v e r y, s=s t r o n g$, and $m=$ medium. Symmetries of the vibrations have been assigned with Schoenfließ symbols.

\begin{tabular}{|c|c|c|c|c|c|c|c|c|}
\hline$\tilde{v}^{\text {Theo }}$ & $I^{\text {Theo }}{ }_{\text {rel }}$ & $\tilde{v}^{\mathrm{HM}}$ & $\Delta \tilde{v}$ HM & $\tilde{v} \tilde{v}^{\text {Expt }}$ & $I_{\text {rel }}^{\text {Expt }}$ & $\tilde{v}$ Ref & $I^{\text {Ref }}$ & Vibration \\
\hline 86.4 & 0.07 & 85.1 & -1.3 & 85.2 & 0.07 & - & - & single Me torsion, $\left\{a^{\prime \prime}\right\}$ \\
\hline 120.5 & 0.08 & 132.9 & 12.4 & - & - & - & - & single Me torsion, $\left\{\mathrm{a}^{\prime \prime}\right\}$ \\
\hline 194.9 & 0.79 & 203.5 & 8.6 & 203.5 & 1.00 & 204 & $v s-a_{1}$ & Me wagging, a" \\
\hline 231.2 & 0.02 & 225.7 & -5.5 & - & - & - & - & Me twisting, a" \\
\hline 259.6 & 0.11 & 276.2 & 16.6 & 277.0 & 0.07 & 278 & $s-a_{1}$ & Me scissoring, $a^{\prime}$ \\
\hline 376.1 & 0.05 & - & - & 403.4 & 0.07 & 402 & $m-b_{2}$ & Me rocking, $a^{\prime}$ \\
\hline 436.4 & 1.00 & 432.2 & -4.2 & 432.3 & 0.52 & - & - & ring bending, a" \\
\hline 496.6 & 0.11 & 511.6 & 15.0 & 488.7 & 0.03 & - & - & ring stretch, $a^{\prime}$ \\
\hline 511.2 & 0.01 & 528.6 & 17.4 & 513.1 & 0.04 & - & - & ring bending, a" \\
\hline 521.3 & 0.01 & 537.4 & 16.0 & 534.5 & 0.03 & - & - & ring stretch, a' \\
\hline
\end{tabular}


Table 7: Theoretical (Theo), Hard Model fit (HM), and present (Expt) [15] and reference (Ref) [2] experimental peak positions $\tilde{v}$ in $\left(\mathrm{cm}^{-1}\right)$, relative intensities $I_{\text {rel }}=T^{2} / T_{\text {max }}^{2}=A / A_{\max }$ wavenumber shifts $\Delta \tilde{v}$, and corresponding vibrations of $p$-xylene in $C_{2 h}$ symmetry. The reference intensities abbreviate with $v=v e r y$ and $s=s t r o n g$. Symmetries of the vibrations have been assigned with Schoenfließ symbols.

\begin{tabular}{|c|c|c|c|c|c|c|c|c|}
\hline$\tilde{v}^{\text {Theo }}$ & $I_{\text {rheo }}$ & $\tilde{v}^{H M}$ & $\Delta \tilde{v}{ }^{H M}$ & $\tilde{v}$ Expt & $I^{\mathrm{Expt}}$ rel & $\tilde{v} \operatorname{Ref}$ & $I_{\text {rel }}^{\text {Ref }}$ & Vibration \\
\hline 77.7 & 0.08 & 78.8 & 1.1 & - & - & - & - & $\begin{array}{l}\text { syn Me torsion }+M e \\
\text { wagging, } a_{u}+b_{u}\end{array}$ \\
\hline 159.5 & 0.05 & 156.2 & -3.3 & 149.0 & 0.43 & 166 & $v s-b_{3 u}$ & syn Me torsion, $a_{u}$ \\
\hline 278.0 & 0.03 & 291.8 & 13.8 & 291.6 & 0.06 & - & - & Me scissoring, $a_{u}$ \\
\hline 404.2 & 0.01 & 412.2 & 8.0 & - & - & - & - & ring bending, $a_{u}$ \\
\hline 471.7 & 1.00 & 483.5 & 11.8 & 483.5 & 1.00 & - & - & ring bending, $b_{u}$ \\
\hline
\end{tabular}

